\title{
Management of pigmented skin lesions in childhood and adolescence
}

Erica B Friedman, Richard A Scolyer, John F Thompson

\section{Background \\ Pigmented skin lesions in childhood and adolescence can be diagnostically challenging. It is important for general practitioners to be aware of the spectrum of benign, atypical and malignant pigmented lesions occurring in these patient groups and of features that should raise concern.}

\section{Objective}

The aims of this article are to assist recognition of high-risk skin lesions encountered in childhood and adolescence and to provide an understanding of the features and management of suspected melanoma in this population.

\section{Discussion}

In children and adolescents, there exist a variety of congenital and acquired naevi and other pigmented skin lesions that can be diagnostically problematic. Additionally, conventional detection criteria for melanoma seen in adults are often not present in children and adolescents, making diagnosis more difficult. Melanoma, if diagnosed in these populations, should be treated at a specialist centre whenever possible.
PIGMENTED SKIN LeSIONS in children and adolescents can present many diagnostic challenges. We describe the clinical features of benign, atypical and malignant pigmented skin lesions in childhood and adolescence to assist the general practitioner (GP) in their diagnosis and management.

\section{Pigmented skin lesions in childhood and adolescence}

The definition of 'childhood' varies in the literature, with some defining childhood as up to 18 years or even 21 years. However, a more widely accepted and useful definition classifies childhood as 'pre-pubertal', ages $0-14$ years, and adolescence as ages $15-19$ years. ${ }^{1,2}$ Pigmented lesions in childhood and adolescence can be either congenital (ie present at birth) or acquired. The latter most often develop in fair-skinned individuals, usually as a result of sun exposure.

\section{Congenital melanocytic naevi} Congenital melanocytic naevi (CMN) are present at birth in approximately 1 in 100 newborns. The risk of malignant transformation for small CMN (Figure 1A) is very low $(<1 \%)$ and most often occurs after puberty. ${ }^{3}$ The necessity to remove small CMN is debatable, but if removal is desired it is generally considered reasonable to wait until adolescence. Giant congenital naevi (GCN), defined as involving $>2 \%$ of the total body surface area (with projected adult size $>20 \mathrm{~cm}$ in diameter), occur much less frequently ( 1 in 20,000 newborns). ${ }^{4}$ GCN are most commonly located on the trunk, have a verrucous surface with irregular pigmentation and are often associated with satellite melanocytic lesions (Figure 1B). GCN may extend into the dermis, subcutaneous tissues and muscle, making surveillance for malignant transformation extremely difficult. The lifetime risk for children born with GCN is difficult to quantify, but a large systematic review reported the development of melanoma in $2 \%$ of children with GCN, most often occurring in GCN $>40 \mathrm{~cm}(74 \%)$ and in those with satellite lesions $(94 \%){ }^{5}$ The highest risk for transformation is during childhood. ${ }^{5}$ Importantly, these melanomas are not limited to the skin, and may develop viscerally or manifest as primary tumours within the central nervous system in association with neurocutaneous melanosis. ${ }^{6}$ The surgical management of GCN is controversial, as prophylactic surgical excision does not eliminate melanoma risk because of deep cutaneous extension of naevus cells, sometimes even extending through the muscular fascia. Lifelong surveillance of patients with GCN is essential.

\section{Acquired melanocytic naevi}

Acquired melanocytic naevi are benign proliferations of melanocytes, thought 
to be clonal in origin, that usually appear in childhood in sun-exposed areas and can increase in size during puberty. Very occasionally, small melanocytic naevi appear in the neonatal period and in the first year or two of life. Although they may have characteristics similar to those of $\mathrm{CMN}$, and are referred to as 'congenital naevus-like naevi', they are not, in fact, congenital.

Though the majority of melanomas across all ages arise de novo, without an associated naevus, ${ }^{7}$ both the size and number of acquired melanocytic naevi are linked to melanoma risk.

\section{Dysplastic naevi}

Dysplastic naevi are acquired atypical pigmented lesions that may display some of the more conventional features of melanomas, namely asymmetry, colour variegation and size $>6 \mathrm{~mm}$, but do not meet the diagnostic threshold for a clinical diagnosis of melanoma (Figure 2A). Histologically, dysplastic naevi show architectural disorder and cytological atypia that are absent in common acquired and congenital naevi, but they lack features of malignancy that occur in melanomas. While dysplastic naevi are potential melanoma precursors, few will actually evolve into melanomas, but their presence defines a population at higher risk for developing melanoma. ${ }^{8}$ As melanoma is more likely to arise de novo than from a pre-existing naevus, prophylactic surgical excision of dysplastic naevi is not recommended, even in this higher-risk population. However, close monitoring is warranted, and surgical excision of changing lesions with a narrow margin is indicated for diagnostic purposes. ${ }^{9}$

\section{Blue naevi}

Blue naevi are an uncommon variant of dermal naevi that can be worrying to the untrained eye. In children or adolescents, blue naevi are typically well-circumscribed, dome-shaped lesions, usually $<10 \mathrm{~mm}$ in diameter, on the scalp, the buttocks and the dorsum of extremities (Figure 2B). Their blue appearance is due to the presence of pigment in the dermis and resulting preferential scattering and reflection of blue versus red light by the skin (the Tyndall effect); this can be confused with the blue-white veil seen on dermoscopic evaluation of some melanomas. ${ }^{10}$ Histologically, blue naevi are composed of pigmented dendritic dermal melanocytes. Rare transformation into melanomas has been reported, most commonly on the scalp. ${ }^{11}$ If clinical suspicion warrants a biopsy, a narrowmargin excision biopsy is recommended to allow for complete histological evaluation.

\section{Naevus spilus}

Naevus spilus may be congenital or acquired. It appears as a collection of darkly pigmented macules and papules on a background of hyperpigmentation that can span up to $20 \mathrm{~cm}$ (Figure 2C). It may be distributed in a dermatomal pattern. Malignant transformation is rare. ${ }^{12}$

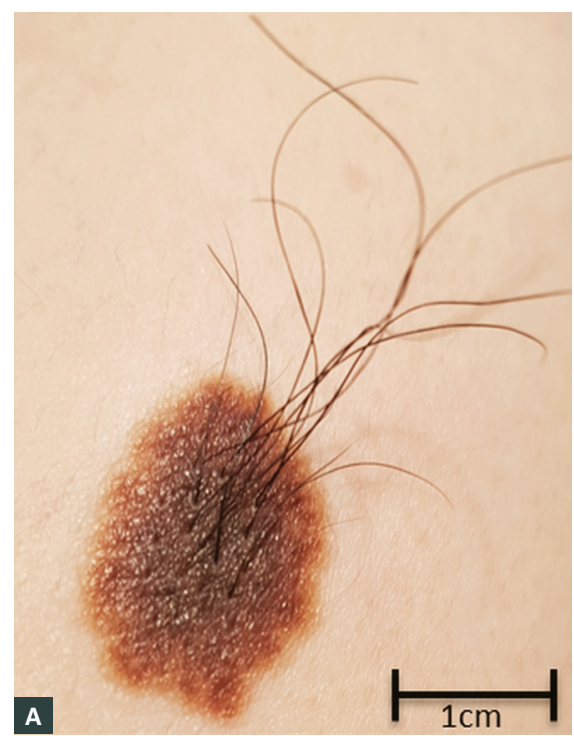

Figure 1. A. Small congenital melanocytic naevus on the lateral shoulder of a male aged 18 years; B. Giant melanocytic naevus on the back of a newborn, involving $>2 \%$ of total body surface area and satellite lesions

Figure 1B courtesy of Dr MA Ansary, Science Photo Library

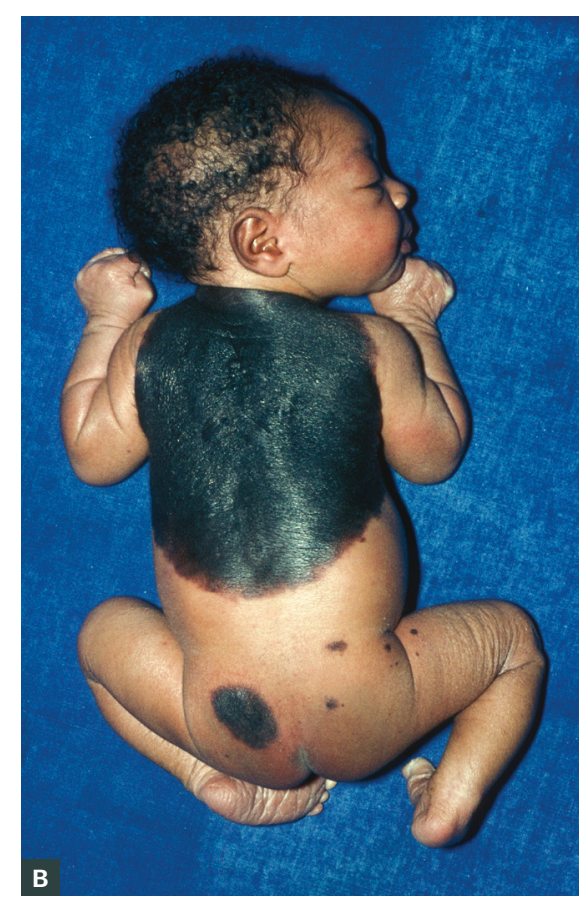

\section{Halo naevi}

Mainly occurring in adolescents, a halo naevus is an acquired (usually compound) naevus that develops a surrounding symmetrical 'halo' of depigmentation (Figure 2D). This can be a source of concern for both parents and the practitioner, as halos can occasionally appear around melanomas, but usually in an asymmetrical fashion. However, halo naevi are benign, and over time (usually within 12-18 months) the central naevus can regress and the depigmented skin regains normal pigmentation. Histologically, a dense lymphocytic infiltrate is seen, associated with a common acquired naevus with bland, mature features. Evaluation of a halo naevus should focus on the central pigmented lesion itself, not on the surrounding halo.

\section{Spitz naevi}

A Spitz naevus is a benign tumour usually appearing in the first decade of life as a solitary, well-circumscribed, pink or pigmented papule $<1 \mathrm{~cm}$ in 
diameter, most often on the head, neck or extremities (Figure 3A). Histologically, there is overlap between features of Spitz naevi and melanomas, making accurate diagnosis difficult. Nevertheless, when the constellation of histological features is present in an appropriate clinical context, Spitz naevi can be confidently diagnosed. Lesions that display some histological features of Spitz naevi but also more concerning atypical features are often referred to as 'atypical Spitzoid tumours' and are categorised as 'melanocytic tumours of uncertain malignant potential' (MelTUMPs).

\section{Pigmented epithelioid melanocytomas}

Pigmented epithelioid melanocytomas (PEMs) most commonly arise in children, adolescents and young adults. PEMs are dermal lesions that may mimic melanoma clinically and pathologically. Histologically, these lesions have infiltrative borders and are composed of heavily pigmented large epithelioid and spindled melanocytes. These lesions frequently spread to regional nodes, but the clinical course is indolent, and PEM is regarded as a borderline or low-grade malignancy. These tumours should be managed similarly to other MelTUMPs (melanocytomas).

\section{MelTUMPs (melanocytomas)}

For some melanocytic tumours that display atypical histological features, it can be difficult or impossible, even for acknowledged experts, to predict biological
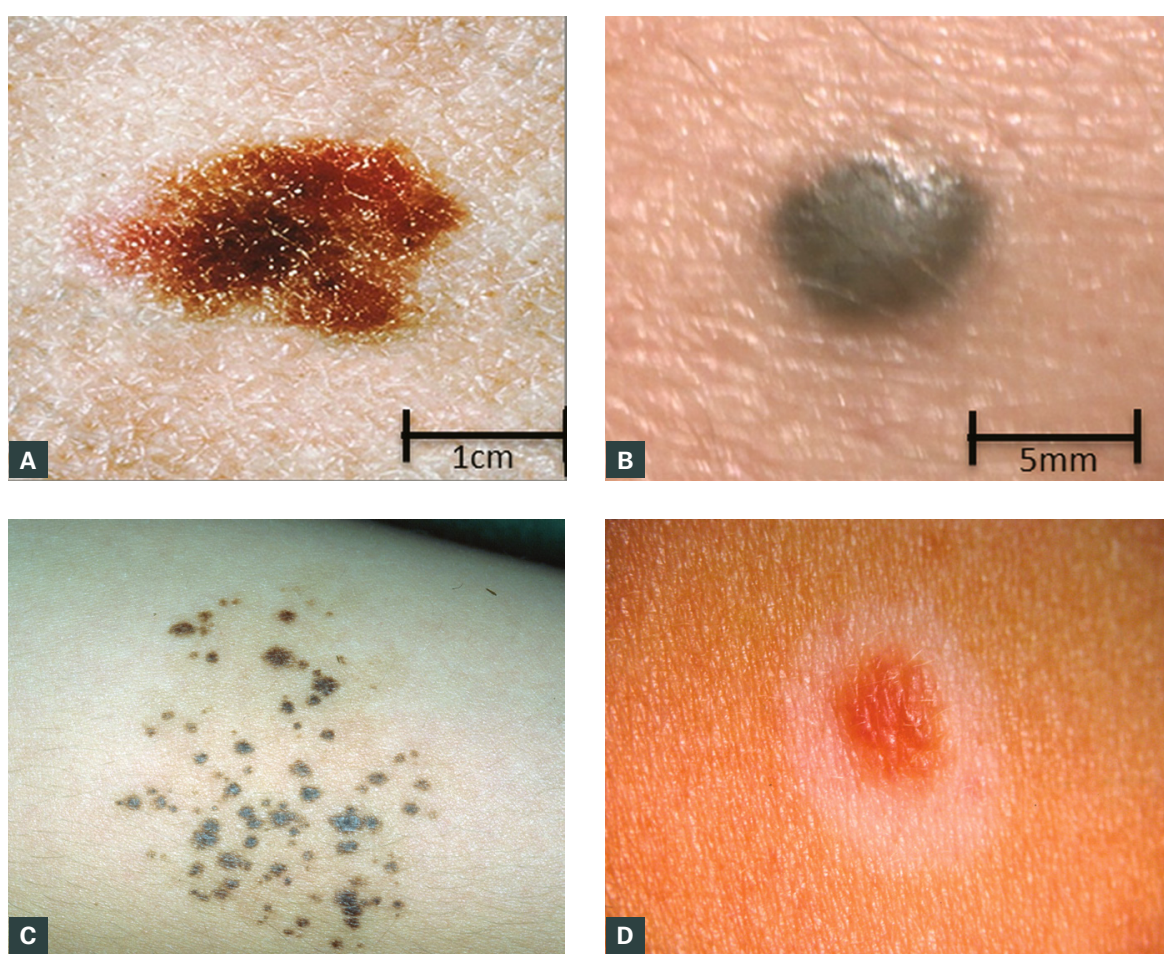

Figure 2. A. Dysplastic naevus on the back of a female aged 16 years, $20 \times 11 \mathrm{~mm}$, demonstrating size $>6 \mathrm{~mm}$, asymmetry and colour variegation that could be mistaken for melanoma; B. Blue naevus, a well-circumscribed, dome-shaped lesion with blue/black colour mimicking melanoma; C. Naevus spillus on the arm of a female aged 15 years, showing darkly pigmented macules on a background of hyperpigmentation; D. Halo naevus, with a symmetrical halo of depigmentation surrounding a compound naevus

Figure 2B courtesy of Dr Jennifer Stein; Figure 2C courtesy of DermisNet; Figure 2D courtesy of DermnetNZ.org behaviour from pathological assessment. Such pigmented lesions in children and adolescents are ultimately designated as 'MelTUMPs' or 'melanocytomas'. Because of the lack of diagnostic certainty, the histology of these lesions should be reviewed by an expert dermatopathologist, and the patients managed at a high-volume referral centre. The lesions should be definitively excised with $5 \mathrm{~mm}$ surgical margins, and the decision to perform a sentinel lymph node (SNL) biopsy made on a case-by-case basis.

\section{Childhood and adolescent melanoma \\ Epidemiology}

Melanoma in childhood is rare, and melanoma in adolescence is uncommon (Figure 4). The reported incidence rates of true childhood (ages 0-14 years) melanoma and adolescent (ages 15-19 years) melanoma in Australia were most recently estimated to be 2.0 per million/year and 24.3 per million/year, respectively ${ }^{13}$

\section{Presentation and diagnosis}

Making the diagnosis of childhood and adolescent melanoma (CAM) can be difficult because patients often do not present with the classical features of adult melanomas. When compared with adults, a higher percentage of young patients with melanoma have a family history of melanoma and atypical naevi, suggesting an underlying genetic origin. However, recent genomic data indicate that ultraviolet (UV) radiation damage still has an important aetiological role in CAM. ${ }^{14} \mathrm{~A}$ high index of suspicion is necessary when evaluating young patients, especially adolescents, who have a phenotypic propensity to develop naevi. ${ }^{15}$ Clinically, melanomas arising in the paediatric population, especially pre-pubertal children, do not always follow the ABCDE (Asymmetry, Borders, Colours, Diameter, Evolving) detection criteria used for adults. ${ }^{7}$ Rather, they often present with amelanotic, papulonodular lesions that lack colour variegation and have arisen de novo (Figure 3B). As a result of their non-conventional appearance, the 
differential diagnosis includes juvenile xanthogranuloma, dermatofibroma, pyogenic granuloma, haemangioma and melanocytoma. Because the conventional ABDCE criteria used in adults are often inadequate in children, alternative ABCDE criteria that could prevent diagnostic delays have been proposed (Table 1).

For suspicious pigmented lesions occurring in children, as in adults, an initial excision biopsy with narrow margins should be performed. This allows histological evaluation of the full lesion. Pathological assessment by an experienced dermatopathologist is recommended, as pigmented lesions, especially with Spitzoid characteristics, can be extremely difficult to characterise as benign or malignant. Additionally, the use of fluorescence in situ hybridisation, comparative genomic hybridisation and gene expression analysis is increasing in an effort to improve characterisation of atypical lesions of uncertain malignant potential. ${ }^{10}$
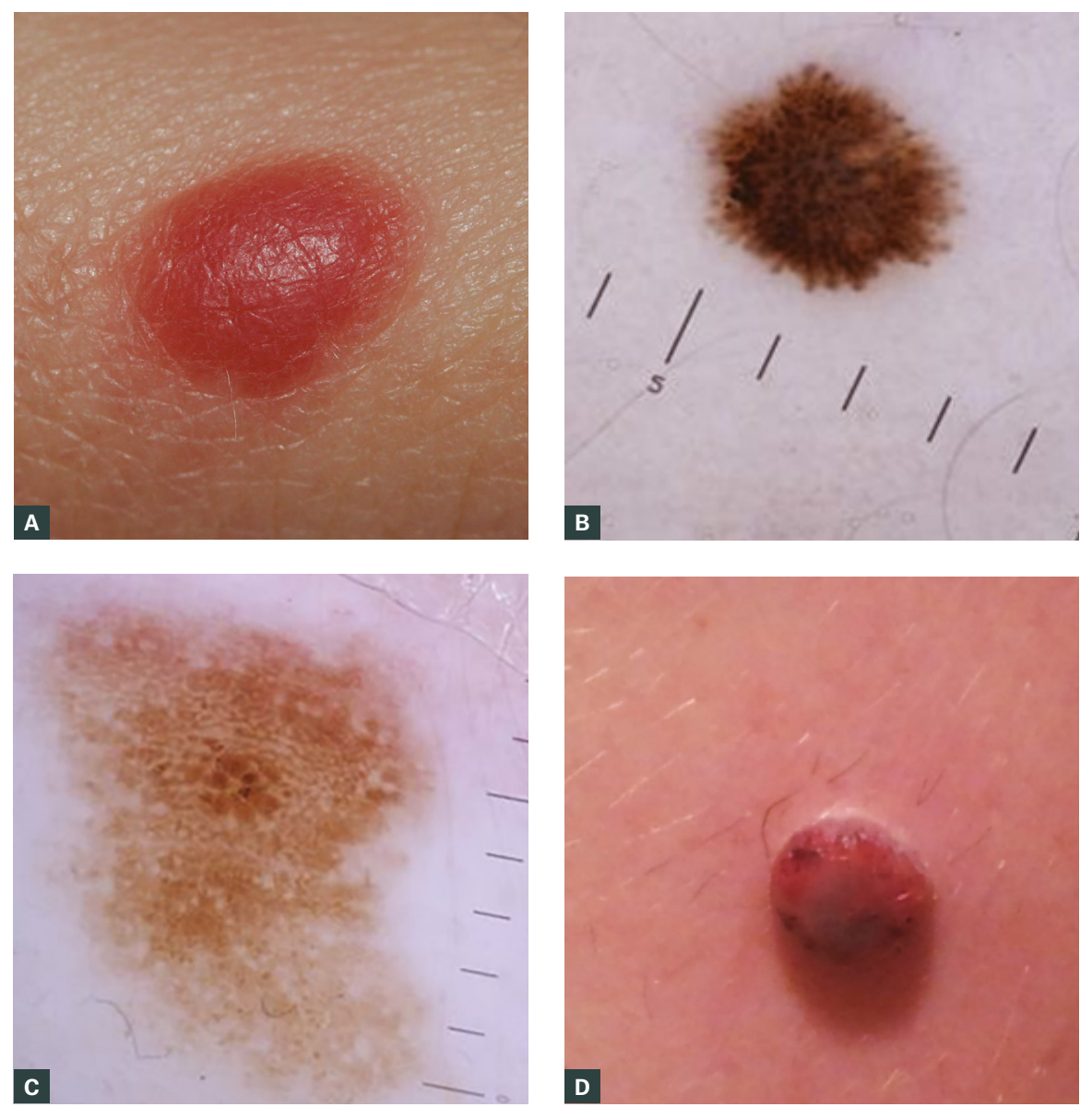

Figure 3. A. A classic Spitz naevus in childhood - a dome-shaped red papule, $<1 \mathrm{~cm}$ in diameter; B. Dermoscopic appearance of a Spitz naevus in a male aged five years, showing a starburst pattern, indicating the radial growth phase of the tumour, central homogenous pigmentation surrounded by pseudopods and finger-like projections regularly and symmetrically distributed at periphery; C. Dermoscopic features of an atypical spitzoid tumor include atypical multicomponent dermoscopic pattern characterised by atypical pigment network, irregular dots and globules, irregular pigmentation and irregular streaks; D. Spitzoid melanoma on the leg of a boy aged 10 years - an amelanotic, nodular lesion that lacks traditional ABCDE diagnostic criteria

Figures $3 B$ and $3 C$ courtesy of Professor Pascale Guitera

\section{Management and prognosis}

Once a diagnosis of melanoma has been made, surgical excision remains the mainstay of treatment. Regardless of the clinical scenario in which a CAM arises, recommended surgical excision margins should parallel those of adults, which are based on Breslow thickness. Consideration of SLN biopsy in children and adolescents is also as in adults, based on the prognostic features of the primary tumour (thickness, ulceration and mitotic rate). Improved outcomes are seen when patients with CAM receive initial surgical care at specialised centres, even when controlling for the stage of melanoma at presentation. ${ }^{16}$

Similarly, children and adolescents who present with clinically-detected regional nodes in the absence of metastatic disease should be treated with lymphadenectomy. Systemic therapy for adjuvant treatment and/or in the metastatic setting should be discussed by a multidisciplinary team and administered at a tertiary referral centre. While children often present with thicker melanomas, ${ }^{17}$ and are more likely to have lymph node metastases, ${ }^{14}$ no major differences in outcomes are found between children/adolescents and adults. ${ }^{1,14,17}$ However, a small retrospective study found that children aged $<10$ years have significantly better survival rates. ${ }^{17}$ Improved outcomes, including five-year overall survival and disease-free survival, are seen when patients with CAM receive initial surgical care at specialised centres, even when controlling for the stage at presentation. ${ }^{16}$

\section{Conclusion}

The assessment and management of pigmented skin lesions in children and adolescents can be challenging. A sound knowledge of the more common pigmented lesions and worrying features is essential for GPs. Early diagnosis and referral of patients with high-risk lesions to an appropriate specialist service is key to improving outcomes.

- Congenital and acquired pigmented lesions in children and adolescents should be identified early and the intensity of surveillance dictated by the risk of malignant transformation. 
- A history of UV exposure, a family history of melanoma, and the presence of dysplastic naevi should alert GPs to a risk of melanoma, even in children and adolescents.

- If suspicious features or changes in pigmented lesions are noted, narrowmargin excision biopsy should be performed, when possible, to allow for complete histological assessment.

- A knowledge of alternative ABCDE diagnostic criteria for melanoma in children and adolescents will help to avoid diagnostic delays.
- Children and adolescents diagnosed with MelTUMPs or melanoma should be referred to specialised centres for management.

\section{Implications for general practice}

The diagnosis and management of pigmented skin lesions in children and adolescents can be challenging. While a variety of pigmented lesions occur in these populations and most are benign, the possibility of melanoma must always be considered because

\section{Table 1. Diagnostic criteria for melanoma in adults compared with children}

\begin{tabular}{lll}
\hline & $\begin{array}{l}\text { Conventional diagnostic criteria } \\
\text { in adults }\end{array}$ & $\begin{array}{l}\text { Proposed diagnostic criteria } \\
\text { in children }\end{array}$ \\
\hline A & Asymmetry & Amelanotic \\
\hline B & Border irregularity & Bleeding or Bump \\
\hline D & Colour variegation & Colourless or Colour uniformity \\
\hline E & Diameter $>6 \mathrm{~mm}$ & De novo appearance, or Diameter $<6 \mathrm{~mm}$ \\
\hline
\end{tabular}

Adapted from Cordoro KM, Gupta D, Frieden IJ, McCalmont T, Kashani-Sabet M. Pediatric melanoma: Results of a large cohort study and proposal for modified ABCD detection criteria for children. J Am Acad Dermatol 2013;68(6):913-25.

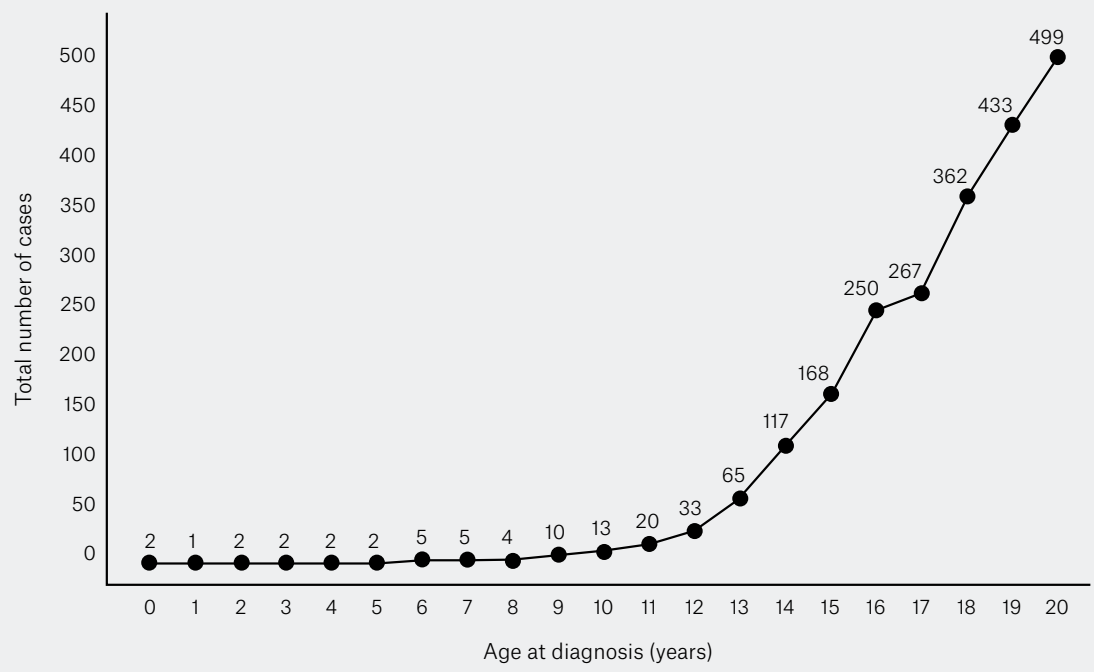

Figure 4. Number of childhood and adolescent melanoma cases in Australia, excluding NSW, from 1982-2014, based on data from the Australian Institute of Health and Welfare Figure courtesy of Dr Serigne Lo a delay in diagnosis can have adverse consequences. It is therefore important for GPs to have a thorough understanding of the more common pigmented lesions in childhood and adolescence, and to be able to recognise suspicious features that should prompt further action.

\section{Authors}

Erica B Friedman MD, MS, Assistant Professor of Surgery, Division of Surgical Oncology, NYU Langone Health, New York, NY, USA

Richard A Scolyer MD, Conjoint Medical Director, Melanoma Institute Australia, University of Sydney, Sydney, NSW; Clinical Professor, Discipline of Pathology, Faculty of Medicine and Health, University of Sydney, Sydney, NSW; Senior Staff Specialist in Anatomical Pathology, Tissue Pathology and Diagnostic Oncology, Royal Prince Alfred Hospital, Sydney, NSW.

John F Thompson MD, Chairman, Australian Melanoma Clinical Practice Guidelines Working Party; Professor of Melanoma and Surgical Oncology, University of Sydney, Sydney, NSW; Surgeon, Royal Prince Alfred Hospital and Melanoma Institute Australia, Sydney, NSW. john.thompson@melanoma.org.au

Competing interests: RS reports membership on the advisory board for Merck Sharp \& Dohme, Novartis, Myriad and NeraCare, outside the submitted work. JT reports membership on the advisory board for and honoraria from GlaxoSmithKline, BMS, Provectus and MSD, as well as travel support from GlaxoSmithKline and Provectus, outside the submitted work.

Funding: None.

Provenance and peer review: Commissioned, externally peer reviewed.

\section{Acknowledgments}

The authors would like to acknowledge and thank Dr Jennifer Stein (NYU Dermatology, New York, NY, USA) for the provision of a clinical image, Dr Serigne Lo (Melanoma Institute Australia, NSW; University of Sydney, Sydney, NSW) for his assistance in the creation of Figure 4 and Kaye Oakley (Melanoma Institute Australia, University of Sydney) for her assistance in manuscript preparation.

\section{References}

1. lannacone MR, Youlden DR, Baade PD, Aitken JF, Green AC. Melanoma incidence trends and survival in adolescents and young adults in Queensland, Australia. Int J Cancer 2015;136(3):603-09. doi: 10.1002/ijc.28956.

2. Baade PD, Youlden DR, Valery PC, et al. Trends in incidence of childhood cancer in Australia, 1983-2006. Br J Cancer 2010;102(3):620-26. doi: 10.1038/sj.bjc.6605503.

3. DermNet NZ. All about the skin. Auckland, New Zealand: DermNet NZ, 2019. Available at www. dermnetnz.org [Accessed 12 June 2019].

4. Castilla EE, da Graça Dutra M, Orioli-Parreiras IM. Epidemiology of congenital pigmented naevi: I. Incidence rates and relative frequencies. $\mathrm{Br} J$ Dermatol 1981;104(3):307-15.

5. Vourc'h-Jourdain M, Martin L, Barbarot S; aRED. Large congenital melanocytic nevi: Therapeutic management and melanoma risk: A systematic review. J Am Acad Dermatol 2013;68(3):493-98. e1-14. doi: 10.1016/j.jaad.2012.09.039. 
6. Balch CM, Houghton AN, Sober AJ, Soong S-J, Atkins MB, Thompson JF. Cutaneous melanoma. 5th edn. Saint Louis, MO: CRC Press, 2009.

7. Cordoro KM, Gupta D, Frieden IJ, McCalmont T, Kashani-Sabet M. Pediatric melanoma: Results of a large cohort study and proposal for modified ABCD detection criteria for children. J Am Acad Dermatol 2013;68(6):913-25. doi: 10.1016/j. jaad.2012.12.953.

8. Novakovic B, Clark WH Jr, Fears TR, Fraser MC, Tucker MA. Melanocytic nevi, dysplastic nevi, and malignant melanoma in children from melanoma-prone families. J Am Acad Dermatol 1995;33(4):631-36. doi: 10.1016/01909622(95)91284-3.

9. Duffy K, Grossman D. The dysplastic nevus: From historical perspective to management in the modern era: Part II. Molecular aspects and clinical management. J Am Acad Dermatol 2012;67(1):19. e1-2; quiz 31-2. doi: 10.1016/j.jaad.2012.03.013.

10. Stretch JR. Melanoma principles \& practice A comprehensive guide. Sydney: Melanoma Institute Australia, 2018.

11. Goldenhersh MA, Savin RC, Barnhill RL, Stenn KS. Malignant blue nevus. Case report and literature review. J Am Acad Dermatol 1988;19(4):712-22.

12. Boot-Bloemen MCT, de Kort WJA,

van der Spek-Keijser LMT, Kukutsch NA. Melanoma in segmental naevus spilus: A case series and literature review. Acta Derm Venereol 2017;97(6):749-50. doi: 10.2340/000155552646.

13. Australian Institute of Health and Welfare. Cancer in adolescents and young adults in Australia. Canberra: AlHW, 2018.

14. Livestro DP, Kaine EM, Michaelson JS, et al. Melanoma in the young: Differences and similarities with adult melanoma: A case-matched controlled analysis. Cancer 2007;110(3):614-24. doi: 10.1002/cncr.22818.

15. Youl P, Aitken J, Hayward N, et al. Melanoma in adolescents: A case-control study of risk factors in Queensland, Australia. Int J Cancer 2002;98(1):92-98. doi: 10.1002/ijc.10117.

16. Freemyer $B$, Hamilton $E$, Warneke $C L$, et al. Treatment outcomes in pediatric melanoma - Are there benefits to specialized care? J Pediatr Surg 2016;51(12):2063-67. doi: 10.1016/j. jpedsurg.2016.09.039.

17. Ferrari A, Bono A, Baldi $M$, et al. Does melanoma behave differently in younger children than in adults? A retrospective study of 33 cases of childhood melanoma from a single institution. Pediatrics 2005;115(3):649-54. doi: 10.1542/ peds.2004-0471. 